В современном профессиональном образовании поиск, разработка и реализация новых ресурсов и механизмов в подготовке конкурентоспособной творческой личности с потребностью непрерывного самосовершенствования и саморазвития осуществляется с учетом дидактического потенциала дисциплин и современных образовательных технологий. С учетом этой позиции, изучая и анализируя многокомпонентный состав профессиональной подготовки, исследователи отмечают педагогический потенциал информационно-коммуникационных технологий и математических дисциплин в формировании профессиональной компетентности, а также навыков самоопределения, самоорганизации и рефлексии. Тем самым, целью статьи является исследование специфики формирования рефлексивной позиции студентов в процессе обучения математики в вузе средствами информационно-коммуникационных технологий. Ведущим методом при этом выступает проектирование методической системы обучения математике в вузе, с включением в нее дидактического инструментария, способствующего повышению интереса обучающихся к предмету, а также развитию навыков самоопределении, самоорганизации и самоанализа. В результате проводимого исследования авторами статьи определены дидактический потенциал информационно-коммуникационных технологий в формировании рефлексивной позиции студентов в процессе обучения математике; методические аспекты включения в процесс обучения математике студентов в вузе разнообразных форм взаимодействия субъектов образовательного процесса посредством использования информационно-коммуникационных технологий; разработана методика «Я познаю себя» с целью формирования рефлексивной позиции студентов.

Ключевые слова: обучение студентов в вузе, рефлексивная позиция, математическая подготовка, формирование рефлексивной позиции студентов, информационно-коммуникационные технологии

Perspectives of Science \& Education. 2018. 6 (36)

International Scientific Electronic Journal

ISSN 2307-2334 (Online)

Available: psejournal.wordpress.com/archive18/18-06/

Accepted: 12 November 2018

Published: 1 January 2019

No. 6 (36). pp. 65-73.

G. I. Simonova, A. V. Konysheva, N. V. Kotryakhov

\title{
Peculiarities of Reflexive Position Formation in Students within Educational Process at the University by Information-and-Communication Technologies
}

In modern professional education, the search, development and implementation of new resources and mechanisms in preparation of competitive creative personality with the demand of sustainable self-improvement and self-development is effected considering didactic potential of disciplines and modern educational techniques. Considering this position, investigating and analyzing the multicomponent structure of professional education, the researches highlight the pedagogical potential of information-andcommunication technologies and mathematical disciplines in professional competence formation, as well as self-identification, self-organization and reflexive skills. Hereby, the article objective is to investigate the specificity of students' reflexive position formation in process of studying mathematics at the university by means of information-and-communication technologies. As leading method in this event acts methodological system development to study mathematics at university, incorporating didactic tools, promoting the interest to the subject development in students, and skills on self-identification, self-organization and self-analysis formation. The conducted by authors investigations resulted in identifying the didactic potential of informationand-communication technologies in students' reflexive position formation in process of studying mathematics; methodological aspects of incorporating into mathematics teaching process for university students various forms of interaction of subjects of educational process by means of use of information-and-communication technologies; the methodology "I perceive myself" has been developed with the purpose to form reflexive position in students.

Key words: students' education at university, reflexive position, mathematical training, reflexive position formation in students, information-and-communication technologies 
процесса активно разрабатывается как зарубежными, так и отечественными исследователями. Основополагающим исследованием явилась работа B.Z. Wulfov, V.N. Harkin [12], раскрывающая феномен рефлексии как педагогический процесс. В работе A. Rath [8] подчеркивается, что формирование рефлексивной позиции студента является ключевым условием его успешного обучения и последующей профессиональной реализации. Отмечается, что процесс формирования рефлексивной позиции и рефлексивного мышлении в целом должен быть обусловлен следующими принципами: личностная направленность процесса обучения (индивидуализация); контекстуальность образовательного процесса; субъективный характер процесса обучения. В этой связи, не менее важным для нашего исследования является работа R.M. Khusainova, S. E. Chirkina \& R.G. Gabdrakhmanova [5], посвященная раскрытию вопроса влиянию индивидуальной образовательной траектории на формировании конкурентоспособной личности.

Вопросы, связанные с исследованием психологических и технологических основ формирования рефлексивной позиции субъектов образовательного процесса, являются дискуссионными и заслуживают отдельного внимания для рассмотрения $[1 ; 6 ; 10]$. В частности, в педагогической науке отсутствует единый подход к формированию рефлексивной позиции студентов. Это, главным образом, определяется многообразием дидактических и технологических средств. X. Lin.,C. Hmelo, C.K.Kinzer, T.J. Secules [6] отмечают, что педагогически обоснованная технология играет значительную роль в развитии и поддержке рефлексии студентов.

В качестве основного дидактического инструментария в формировании рефлексивной позиции мы рассматриваем информационнокоммуникационные технологии. Специфика образовательного процесса с внедрением новых технологий представлена в работах В.C. Nelson [7], C. Dede [2], V. Geiger, N. Calder, H. Tan, E. Loong, J. Miller, K. Larkin [4]. Указанные исследования представляет интерес для нас, так как авторами подробно описан процесс влияния цифровых технологий на компоненты педагогического процесса, когнитивные структуры личности, ее профессиональное становление.

В работах J.R. Wang, S.W. Lin [11] и L.A. Yuen Lie Lim [13] исследуются факторы влияющие на формировании рефлексивного мышления и рефлексивной позиции. В частности, в исследовании L.A. Yuen Lie Lim [13] указывается эффективность проблемного обучения в процессе формирования рефлексивного мышления студентов. Такой подход к исследуемой проблеме позволил нам определить систему проблемных задач - как ключевое условие в формировании рефлексивной позиции субъектов образовательного процесса. 
Педагогический потенциал нового инструментария в формировании конкурентоспособного специалиста с высокой готовностью к профессиональной деятельности описан в исследовании M. Ryan [9]. В этом вопросе проблема формирования рефлексивной позиции является ключевой. Автором выделен единый принцип реализации поставленной задачи - принцип интеграции и управляемости.

В отечественной педагогической теории и практики нами были проанализированы вопросы, связанные с использованием педагогического потенциала учебных дисциплин в формировании рефлексивной позиции студентов [14], вопросы, раскрывающие педагогическое сопровождение и возможности использования интерактивных технологий обучения [18]. Исследователи, не смотря на специфику раскрытия рассматриваемого вопроса, сходятся во мнении, что рефлексивная позиция студента, является ключевым компонентом формирования его профессиональной готовности. Схожая позиция отражена в работе авторов, описывающих модель высшего профессионального образования [3]. Особый интерес для нашего исследования, представляют работы, раскрывающие дидактический потенциал математики в формировании рефлексивной позиции студентов [21]. Автором отмечается, что в качестве ключевых приемов, активизирующих механизмы рефлексии в процессе обучения математике, следует рассматривать возможность диалогового взаимодействия (как очного, так и виртуального), возможность визуализации учебного материала.

Рассматривая последний аспект, заметим, что в работах исследователей он в большей степени раскрывается через призму индивидуализации. Так, например, в трудах В.А. Красильниковой [17] доказывается взаимосвязь возможности учета индивидуальных характеристик и уровня мотивации и самоорганизации обучающихся. Это является особенно важным при изучении математики, так как студентам с различными уровнем подготовки и индивидуально-личностными особенностями требуется разное количество времени для восприятия и осмысления информации, представленной в буквенно-цифровой и графической формах. По мнению И. Ю. Соколовой и Г. П. Кабанова [20], именно возможность учета когнитивных стилей - стилей восприятия и переработки информации оказывает значительное влияние на эффективность процесса обучения. Это объясняется тем, что знание ведущего канала восприятия информации и адаптация обучающего материала к индивидуально-личностным особенностям студентов позволяет обеспечивать состояние их психологической и физической комфортности. Следовательно, оказывает непосредственное влияние на формирование рефлексивной позиции. Кроме того, доказывается, что предъявление учебной информации с уче- том функциональной специализации полушарий головного мозга является одним из педагогических условий дифференциации и индивидуализации обучения, и, следовательно, ведет к его совершенствованию. Близкая позиция изложена И. Н. Фроловым [22].

Следует подчеркнуть, что использование информационно-коммуникационных технологий в процессе обучения математике способствуют формированию индивидуальной образовательной технологии студентов. Проблема формирования индивидуальных образовательных траекторий отражена в работах А. В. Хуторского [23], И. С. Якиманской [24] и др. Исследователи выделяют различные подходы к определению сущности рассматриваемого понятия. Так, например, А. В. Хуторской [23] определяет его как персональный путь реализации личностного потенциала; ряд исследователей характеризуют как процесс и результат индивидуального выбора студентом содержания, уровня и пути получения образования посредством осуществления выбора. В психолого-педагогических работах выделяется два направления выбора - горизонтальное и вертикальное. Горизонтальное - обусловливает выбор содержания, форм организации образовательной деятельности. Вертикальное направление обеспечивает выбор оптимального уровня образования. С. И. Осипова, Т. В. Соловьева [19] подчеркивают, что выбор является действием, придающим всей деятельности целенаправленность.

Резюмируя вышесказанное, заключаем, что в большинстве источников, посвященных исследуемой проблематике описывается либо обобщенные теории по формировании рефлексивной позиции студентов в процессе обучения математике средствами информационно-коммуникационных технологий, либо крайне узко специализированные вопросы. Такой подход актуализирует задачу разработки методического сопровождения формирования рефлексивной позиции студентов вузе средствами информационно-коммуникационных технологий в процессе обучения математике.

Материалы и методы

\section{Диагностика уровня сформированности рефлексивной позиции студентов}

Экспериментальная работа была осуществлена на базе Федерального государственного бюджетного образовательного учреждения высшего образования «Вятский государственный университет» посредством ведения опытного преподавания математики для студентов химического и электротехнического факультетов (429 человек).

Теоретическое осмысление сущности понятия «рефлексивная позиция» явилось определяющим в выборе и проведении диагностических 
методик, направленных на выявление уровня сформированности рефлексивной позиции студентов. Нами исследовались такие параметры, как самоорганизация и рефлексивные умения студентов. Указанная позиция была обоснована тем, что эффективность учебно-познавательной деятельности студентов взаимосвязана с уровнем их самоорганизации. В качестве приоритетной задачи, решаемой в этом направлении может быть выделена следующая: сформировать у студентов вполне осознанное и достаточно сложное по своей структуре действие организации времени жизни, а именно: смысловое планирование - выделение целей, подцелей, задач собственной деятельности с точки зрения их смысловой значимости для личности, упорядочения их по степени важности; текущий контроль порядка решаемых задач, учета темпа, ритма, затрат времени на выполнение всех задач и целей; вероятностное прогнозирование - соотнесение ближайших и отдаленных задач во временной перспективе дня, недели, месяца и т.д. При этом исследователи указанной проблематики подчеркивают, что «действие организации времени жизни» неотделимо от понятия "самоорганизация», «самоопределение», «самоанализ», «рефлексия».

С целью изучения уровня самоорганизации студентов нами применялся опросник А. Д. Ишкова [15] «Диагностика особенностей самоорганизации». В ходе исследования нами были получены следующие данные: преобладающее большинство студентов продемонстрировало низкий уровень сформированности умений и навыков самоорганизации; средний уровень был выявлен у 35,5\% респондентов; высокий уровень - у 25,2\% от общего числа студентов. Отметим, что студенты с низким уровнем самоорганизации демонстрируют неосознанный выбор профессии, слабую мотивацию к деятельности, отсутствие систематичности и последовательности в организации учебно-познавательной деятельности, низкую самооценку в достижении поставленной цели, отсутствие навыков осуществления рефлексии. Студенты второй группы характеризуются осознанным выбором профессии, сформированными навыками планирования деятельности и анализа ее результатов. В то же время испытывают затруднения при осуществлении самооценки и рефлексии. Студенты с высоким уровнем самоорганизации характеризуются следующими показателями: адекватный выбор профессии, высокая мотивация, выраженные волевые черты, достаточно высокая адекватная самооценка, целеустремленность, организованность, высокая успеваемость, уверенность в себе, сформированные навыки осуществления рефлексии.Полученные данные в ходе диагностики явились значимыми для нас. В первую очередь, это связано с взаимообусловленностью самоорганизации с рефлексивными процессами.
По мнению ряда исследователей формирование и развитие рефлексии у студентов будет способствовать их выходу на рефлексивную позицию учебно-познавательной деятельности, обеспечит включения собственного «Я» в данный процесс.

Таким образом, принимая во внимание тот факт, что важным структурным элементом самоорганизации является уровень сформированности рефлексивной позиции, обратимся к его анализу. С этой целью мы использовали методику А. В. Карпова [16]. В ходе исследования нами были получены следующие данные: 31,5 \% опрошенных продемонстрировали низкий уровень сформированности рефлексивных умений; 48,3\% студентов - средний уровень; высокий - 20, 2\% респондентов. Анализ данных, полученных нами в ходе проведения двух диагностических методик, актуализировал поиск решения проблемы о формировании рефлексивной позиции студентов. С этой целью нами была спроектирована методическая система обучения математике с учетом дидактического потенциала информационно-коммуникационных технологий.

\section{Проектирование методической системы обучения математике с учетом дидактического потенциала информационно- коммуникационных технологий}

В основу проектирование методической системы обучения математике нами были заложены следующие принципы: системности, технологичности, управляемости и интегративности. Методическая система обучения математике включает взаимосвязанные компоненты (целевой, содержательный, технологический), определяющие логику взаимодействия субъектов образовательного процесса. На первом этапе были определены теоретические основания проектирования методической системы с учетом специфики математической подготовки студентов; на втором этапе происходила разработка содержания методической системы, корректировка системы задания с учетом направленности - формирования рефлексивной позиции студента. На третьем этапе разрабатывался технологический инструментарий с учетом его дидактического потенциала, в том числе и для расширения форм и структуры взаимодействия субъектов образовательного процесса. Нами были структурированы этапы взаимодействия субъектов по форме организации учебно-познавательной деятельности, а именно лекция, видеолекция, вебинар, форум, чат, семинар. В рамках данного взаимодействия происходит решение задач («задачи-алгоритмы», «задачи-исследования», творческие задачи, работа с тестами, тренажерами, анкетами, электронными справочниками и внешними открытыми ресурсами сети-Интернет). При этом следует заметить, что логика про- 
хождения этапов для большинства студентов в силу индивидуально-личностных особенностей не является последовательной, и она может но- сить циклический характер. Логика организации учебно-познавательной деятельности студентов представлена в таблице 1.

Таблица 1

\section{Логика организации учебно-познавательной деятельности студентов}

\begin{tabular}{|c|c|c|c|c|c|}
\hline $\begin{array}{c}\text { № } \\
\text { этапа }\end{array}$ & Содержание & $\begin{array}{c}\text { Форма } \\
\text { организа- } \\
\text { ции }\end{array}$ & Описание & $\begin{array}{l}\text { Тип вза- } \\
\text { имодей- } \\
\text { ствия }\end{array}$ & $\begin{array}{c}\text { Реализация возмож- } \\
\text { ностей информацион- } \\
\text { но-коммуникационных } \\
\text { технологий }\end{array}$ \\
\hline 1 & $\begin{array}{l}\text { Изложение но- } \\
\text { вого материала }\end{array}$ & лекция & $\begin{array}{l}\text { постановка проблемных вопросов, } \\
\text { поиск ответов на которые позволяет } \\
\text { выявить противоречия и подвести } \\
\text { под решение определенных класса } \\
\text { задач с использованием математиче- } \\
\text { ского аппарата; излагаются основные } \\
\text { теоретические сведения курса }\end{array}$ & $\begin{array}{l}\text { комбини- } \\
\text { рованный }\end{array}$ & $\begin{array}{l}\text { интеграция содержания } \\
\text { математики с элемента- } \\
\text { ми профессионального } \\
\text { знания, обеспечение } \\
\text { непрерывного педагоги- } \\
\text { ческого сопровождения } \\
\text { аудиторной и внеаудитор- } \\
\text { ной работы студентов }\end{array}$ \\
\hline 2 & $\begin{array}{l}\text { Актуализация } \\
\text { материала, } \\
\text { систематиза- } \\
\text { ция знаний, } \\
\text { совместный } \\
\text { поиск решения } \\
\text { проблемных } \\
\text { ситуаций }\end{array}$ & $\begin{array}{c}\text { видеолек- } \\
\text { ция, } \\
\text { вебинар }\end{array}$ & $\begin{array}{l}\text { реализуется через организованный } \\
\text { диалог, происходит решение про- } \\
\text { блемных ситуаций, осуществляется } \\
\text { демонстрация ключевых функций } \\
\text { и область применения изучаемых } \\
\text { объектов, устанавливаются при- } \\
\text { чинно-следственные связи с ранее } \\
\text { изученными темами }\end{array}$ & $\begin{array}{c}\text { виртуаль- } \\
\text { ный }\end{array}$ & $\begin{array}{l}\text { интеграция содержания } \\
\text { математики с элементами } \\
\text { профессионального зна- } \\
\text { ния, основанная на общ- } \\
\text { ности изучаемых понятий, } \\
\text { их межпредметных связях; } \\
\text { методов, форм и средств } \\
\text { обучения }\end{array}$ \\
\hline 3 & $\begin{array}{l}\text { Совместное } \\
\text { обсуждение } \\
\text { выдвинутых ги- } \\
\text { потез, сложных } \\
\text { вопросов }\end{array}$ & форум,чат & $\begin{array}{l}\text { организуется посредством оказания } \\
\text { помощи в овладении определенным } \\
\text { методом решения задач или работы } \\
\text { над теоретическим материалом } \\
\text { (консультационный чат); обсуждение } \\
\text { проблемных вопросов }\end{array}$ & $\begin{array}{c}\text { виртуаль- } \\
\text { ный }\end{array}$ & $\begin{array}{l}\text { осуществление оператив- } \\
\text { ной обратной связи; обе- } \\
\text { спечение непрерывного } \\
\text { педагогического сопро- } \\
\text { вождения аудиторной и } \\
\text { внеаудиторной работы } \\
\text { студентов }\end{array}$ \\
\hline 4 & $\begin{array}{l}\text { Решение задач } \\
\text { первого уровня } \\
\text { сложности («за- } \\
\text { дачи-алгорит- } \\
\text { мы») }\end{array}$ & $\begin{array}{c}\text { Самосто- } \\
\text { ятельная } \\
\text { работа }\end{array}$ & $\begin{array}{l}\text { решение задач первого уровня слож- } \\
\text { ности, направленных на воспроиз- } \\
\text { ведение правил, формул, действий } \\
\text { осуществляемых по образцу, форми- } \\
\text { рование умений }\end{array}$ & $\begin{array}{l}\text { комбини- } \\
\text { рованный }\end{array}$ & $\begin{array}{l}\text { учет индивидуаль- } \\
\text { ных характеристик } \\
\text { студентов;создание } \\
\text { симулирующих ситуаций } \\
\text { (ситуаций успеха, введение } \\
\text { элементов состязательно- } \\
\text { сти и др.) }\end{array}$ \\
\hline 5 & $\begin{array}{l}\text { Проведение } \\
\text { on-line тестиро- } \\
\text { вания, диагно- } \\
\text { стики, работа с } \\
\text { тренажерами }\end{array}$ & $\begin{array}{l}\text { работа с } \\
\text { электрон- } \\
\text { ными } \\
\text { ресурсами }\end{array}$ & $\begin{array}{l}\text { осуществляется работа с электрон- } \\
\text { ными ресурсами с целью систе- } \\
\text { матизации знаний и закрепления } \\
\text { навыков решения задач, проводится } \\
\text { диагностика для выявления индиви- } \\
\text { дуально-личностных особенностей } \\
\text { и формирования индивидуальных } \\
\text { образовательных траекторий на по- } \\
\text { следующих этапах обучения }\end{array}$ & $\begin{array}{l}\text { комбини- } \\
\text { рованный }\end{array}$ & $\begin{array}{l}\text { учет психофизиологи- } \\
\text { ческих особенностей и } \\
\text { индивидуальных характе- } \\
\text { ристик студентов; создание } \\
\text { симулирующих ситуаций } \\
\text { (ситуаций успеха, введение } \\
\text { элементов состязатель- } \\
\text { ности и др.); обеспечение } \\
\text { атмосферы сотрудничества } \\
\text { и диалогового взаимодей- } \\
\text { ствия }\end{array}$ \\
\hline 6 & $\begin{array}{l}\text { решение за- } \\
\text { дач второго и } \\
\text { третьего уровня } \\
\text { сложности («за- } \\
\text { дачи-исследова- } \\
\text { ния», творче- } \\
\text { ские задачи) }\end{array}$ & семинар & $\begin{array}{l}\text { проводится с целью выявления } \\
\text { «пробелов» в знаниях, полученных } \\
\text { на предыдущих этапах, проверки } \\
\text { сформированности умений реше- } \\
\text { ния задач репродуктивного уровня; } \\
\text { создаются условия для индивиду- } \\
\text { альной работы каждого студента с } \\
\text { целью формирования аналитических } \\
\text { и исследовательских способностей, } \\
\text { навыков моделирования процессов, } \\
\text { интерпретации и оценки данных }\end{array}$ & $\begin{array}{l}\text { комбини- } \\
\text { рованый }\end{array}$ & $\begin{array}{l}\text { обеспечение атмосферы } \\
\text { сотрудничества и диалого- } \\
\text { вого взаимодействия; осу- } \\
\text { ществление оперативной } \\
\text { обратной связи; обеспече- } \\
\text { ние непрерывного педаго- } \\
\text { гического сопровождения } \\
\text { аудиторной и внеаудитор- } \\
\text { ной работы студентов. }\end{array}$ \\
\hline
\end{tabular}


В частности, для реализации четвертого этапа необходимо обратиться к первому и третьему этапам.

Как наиболее сложный и ключевой шестой этап требует усвоения и систематизации полученной информации, следовательно, актуализует переход от четвертого к третьему, второму и перовому этапам. К пятому этапу можно обратиться сразу после первого с целью получения необходимой информации для формирования индивидуальных образовательных траекторий. Это необходимо с целью актуализации уже имеющихся способов решения задач, а также рассмотрения теоретических вопросов, вызвавших у студентов наибольшие затруднения.

Возможность выбора, нелинейный характер представления учебного материала обеспечивает изучение курса с учетом индивидуально-личностных характеристик студентов, способствует формированию рефлексивной позиции студентов. «Зацикливание» последовательности прохождения этапов от шестого к первому происходит на основании логики изучения выделенных тематических линий дисциплины.

\section{Методика формирования рефлексивной позиции студентов в процессе обучения математике средствами информационно- коммуникационных технологий}

Целевое назначение методики формирования рефлексивной позиции студентов в процессе обучения математике средствами информационно-коммуникационных технологий детализируется следующими задачами: выявление уровня мотивации к изучению математики и получению будущей профессии; определение «начального» уровня математической подготовки студентов; выявление "барьерных» механизмов изучения математики; определение индивидуальной принадлежности к типу когнитивной модальности; диагностирование сформированности навыков самоорганизации, самоконтроля и рефлексивных умений. Разработанная методика представлена тремя модулями: информационный, проектировочный, рефлексивный.

Информационный модуль направлен на информирование обучающихся о специфике организации собственной деятельности и планирования времени; содержит задания, направленные на формирования навыков самоорганизации студентов. Проектировочный модуль включает практико-ориентированные задания; направлен на развитие логического и рефлексивного мышления студентов. Рефлексивный блок обеспечивает формирование и корректировку рефлексивной позиции студентов; направлен на самоанализ и саморазвитие личности. Разработанная методика предполагает очное и виртуальное взаимодействие субъектов образовательного процесса.

Результаты исследования

\section{Результаты апробации методики формирования рефлексивной позиции студентов в процессе обучения математике}

Для обоснования эффективности разработанной методики нами были сформированы контрольная и экспериментальная группы. В заключении, на итоговом этапе эксперимента, после апробации всех этапов разработанной методики в экспериментальной группе, нами была проведена итоговая диагностики с целью выявления динамики в процессе формирования рефлексивной позиции студентов средствами информационно-коммуникационных технологий при изучении математики.

Для этого нами была осуществлена проверка и обоснование однородности выборок контрольной и экспериментальной групп с помощью многофункционального критерия Фишера. По результатам исследования мы получили, что «Фи" критическое.

«Фи" вательно с 95\% достоверностью можно утверждать, что не имеется статистически достоверных различий между процентными долями двух выборок по уровню сформированности рефлексивной позиции на начало экспериментальной работы. Таким образом, вышеизложенное теоретическое обоснование позволяет сделать вывод об однородности выборок контрольной и экспериментальной групп.

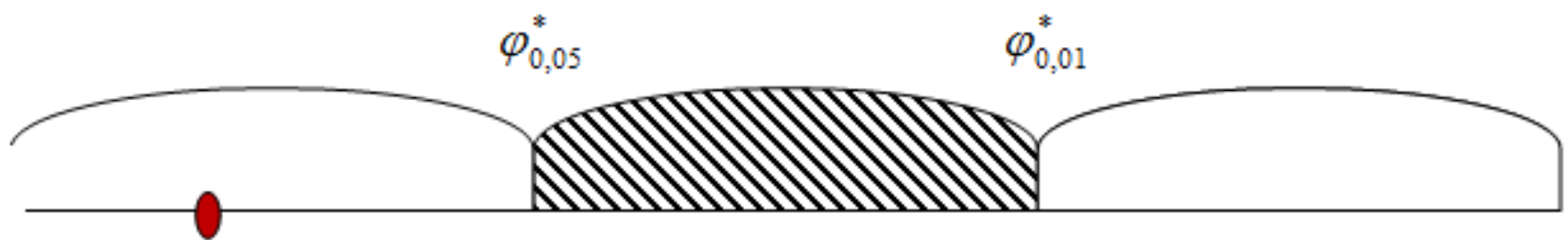

$$
\varphi_{\text {zin }}=0,662 \quad 1.64 \quad 2.31
$$

Рис. 1 Ось значимости.

Критические значения многофункционального критерия Фишера «Фи» ${ }_{0,05}=1,64$ и «Фи» ${ }_{0,01}=2,31$ 
Анализируя уровень сформированности рефлексивных умений на констатирующем и контрольном этапах эксперимента, отметим, что выявлена тенденция изменения в положительную сторону, а именно: уменьшение количества студентов с низким уровнем данного показателя $(19,6 \%)$ и увеличение числа студентов со средним (50,5 \%) и высоким уровнем (29,9\%).

Подчеркнем, что увеличение количества обучающихся, продемонстрировавших высокий и средний уровень сформированности рефлексивной позиции достигнуто посредством предоставления права самостоятельного выбора траектории обучения с ее последующей корректировкой; активизации учебно-познавательной деятельности путем обеспечения профессиональной направленности обучения, использования информационно-коммуникационных технологий. Проведя расчет параметров углового преобразования Фишера для рефлексивного критерия, нами были получены следующие данные: процентная доля студентов с высоким уровнем проявления исследуемого критерия в экспериментальной группе составляет 80,4\%, в контрольной группе - 70,7\%. Исходя из расчетов: «Фи" значение попало в зону "значимости различий» мы можем утверждать на 5\% уровне значимости, что на конец экспериментальной работы в экспериментальной и контрольной группах имеются достоверные различия процентных долей сту- дентов со средним и высоким уровнем сформированности рефлексивной позиции.

Заключение

В ходе проведенного исследования нами была разработана единая методическая система, включающая этапы обучения математике в вузе, сопряженные разнообразными формами диалогового взаимодействия субъектов образовательного процесса посредством информационно-коммуникационных технологий. Разработана методика «я познаю себя» с целью формирования рефлексивной позиции студентов в процессе обучения математике

Выявлен потенциал информационно-коммуникационных технологий в формировании рефлексивной позиции студентов посредством реализации методики «Я познаю себя»

Проведенное нами исследование имеет прогностическое значение, так как создает реальные предпосылки для дальнейшего научного поиска при исследовании специфики внедрения информационно-коммуникационных технологий в образовательную практику с учетом развития информационно-технологических характеристик современного общества, гибкости и сменяющихся ориентиров Федеральных государственных образовательных стандартов, усложнения наукоемких и технологических сфер производства.

1. Biktagirova G.F., Valeeva R.A. Development of the teachers' pedagogical reflection. Life Science Journal, 2014, no. 11(9). p. 60.

2. Dede C. Theoretical Perspectives Influencing the Use of Information Technology in Teaching and Learning. International Handbook of Information Technology in Primary and Secondary Education, Vol. 20. Springer, Boston, MA. 2008. pp. 43-62.

3. Ganieva Y. N., Azitova G. S., Chernova Y. A., Yakovleva I. G., Shaidullina A. R., Sadovaya, V. V.. Model of High School Students Professional Education. Life Science Journal, 2014, no. 11(8s). pp. 1097-8135.

4. Geiger V., Calder N., Tan H., Loong E., Miller J., Larkin K. Transformations of Teaching and Learning Through Digital Technologies. Research in Mathematics Education in Australasia 2012-2015. Springer, Singapore. 2016. pp. 255280.

5. Khusainova R.M., Chirkina S. E., Gabdrakhmanova R.G. The Role of the Reflective Activity of Students in Individual Educational Traectory. Review of European Studies,2015, vol. 7, no. 5. 2015. pp.146-152.

6. Lin, X., Hmelo, C., Kinzer, C.K. et al. Designing technology to support reflection. Educational Technology Research and Development, 1999, volume 47, Issue 3, pp. 43-62. URL: https://doi.org/10.1007/BF02299633

7. Nelson, B.C. Exploring the Use of Individualize, Reflective Guidance In an Educational Multi-User Virtual Environment. Journal of Science Education and Technology, 2007, Volume 16, Issue 1, pp. 83-97.

8. Rath A. Reflective Practice as Conscious Geometry: Portfolios as a Tool for Sponsoring, Scaffolding and Assessing Reflective Inquiry in Learning to Teach. Handbook of Reflection and Reflective Inquiry. Springer, Boston, MA. 2010. pp. 489-515.

9. Ryan M. Sustainable Pedagogical Change for Embedding Reflective Learning Across Higher Education Programs. Teaching Reflective Learning in Higher Education. 2015. pp. 213-227.

10. Semenov I. N. Reflective consciousness and the intuitive creative act. Psychology questions, 2011. no. 6, pp. 152155.

11. Wang J.R., Lin S.W. Examining Reflective Thinking: A Study of Changes in Methods Students' Conceptions and Understandings of Inquiry Teaching. International Journal of Science and Mathematics Education, 2008, volume 6, Issue 3, pp. 459-479.

12. Wulfov B. Z., Harkin, V. N. Pedagogy of reflection (p. 111). Moscow: IChP “Publisher Master". 1995.

13. Yuen Lie Lim L.A. A comparison of students' reflective thinking across different years in a problem-based learning environment. Instructional Science, 2011, Volume 39, Issue 2, pp. 171-188.

14. Багдай Е.В. Формирование рефлексивной позиции студента в процессе изучения гуманитарных дисциплин: 
дис. ... канд. пед. наук. Киров, 2007. 166 с.

15. Ишков А.Д. Учебная деятельность студента: психологические факторы успешности: монография. Москва: Издательство АСВ. 2004. 556 с.

16. Карпов А.В. Рефлексивность как психическое свойство и методика ее диагностики. Психологический журнал. 2003. Том 24. № 5. С. 45-57.

17. Красильникова В.А. Теория и технологии компьютерного обучения и тестирования: монография. Москва: Дом педагогики, ИПК гОУОГУ. 2009. 333 с.

18. Марущак И.П. Педагогическое сопровождение процесса формирования рефлексивной позиции студентов вузов посредством интерактивных технологий обучения: дис. ... канд. пед. наук. Тверь, 2012. 223 с.

19. Осипова С.И. Проектирование студентом индивидуальной образовательной траектории в условиях информатизации образования: монография. Москва: ИНФРА . Красноярск: Сиб. Федер. ун-т. 2013.140 с.

20. Соколова И.Ю., Кабанов Г.П. Качество подготовки специалистов в техническом вузе и технологии обучения : учебное пособие для педагогов, аспирантов, магистрантов. Томск: Изд-во ТГпУ. 2003.203 с.

21. Феклушкина А.В. Формирование рефлексивной деятельности студентов в процессе изучения математики. Личность, семья, общество: вопросы педагогики и психологии. Часть 2. 2011

22. Фролов И.Н. E-learning как форма организации учебного процесса в XXI веке. Информатика и образование. 2009. № 2. С. 109-110.

23. Хуторской А.В. Педагогическая инноватика: учеб. пособие для студ. высших учеб. заведений. Москва: Издательский центр «Академия». 2010. 256 с.

24. Якиманская И.С. Психологические основы математического образования : учеб. пособие для студ. пед. вузов. Москва: Издательский центр «Академия». 2004. 320 с.

REFERENCES

1. Biktagirova G.F., Valeeva R.A. Development of the teachers' pedagogical reflection. Life Science Journal, 2014, no. 11(9). p. 60.

2. Dede C. Theoretical Perspectives Influencing the Use of Information Technology in Teaching and Learning. International Handbook of Information Technology in Primary and Secondary Education, Vol. 20. Springer, Boston, MA. 2008. pp. 43-62.

3. Ganieva Y. N., Azitova G. S., Chernova Y. A., Yakovleva I. G., Shaidullina A. R., Sadovaya, V. V.. Model of High School Students Professional Education. Life Science Journal, 2014, no. 11(8s). pp. 1097-8135.

4. Geiger V., Calder N., Tan H., Loong E., Miller J., Larkin K. Transformations of Teaching and Learning Through Digital Technologies. Research in Mathematics Education in Australasia 2012-2015. Springer, Singapore. 2016. pp. 255280.

5. Khusainova R.M., Chirkina S. E., Gabdrakhmanova R.G. The Role of the Reflective Activity of Students in Individual Educational Traectory. Review of European Studies, 2015, vol. 7, no. 5. 2015. pp.146-152.

6. Lin, X., Hmelo, C., Kinzer, C.K. et al. Designing technology to support reflection. Educational Technology Research and Development, 1999, volume 47, Issue 3, pp. 43-62. URL: https://doi.org/10.1007/BF02299633

7. Nelson, B.C. Exploring the Use of Individualize, Reflective Guidance In an Educational Multi-User Virtual Environment. Journal of Science Education and Technology, 2007, Volume 16, Issue 1, pp. 83-97.

8. Rath A. Reflective Practice as Conscious Geometry: Portfolios as a Tool for Sponsoring, Scaffolding and Assessing Reflective Inquiry in Learning to Teach. Handbook of Reflection and Reflective Inquiry. Springer, Boston, MA. 2010. pp. 489-515.

9. Ryan M. Sustainable Pedagogical Change for Embedding Reflective Learning Across Higher Education Programs. Teaching Reflective Learning in Higher Education. 2015. pp. 213-227.

10. Semenov I. N. Reflective consciousness and the intuitive creative act. Voprosy psychologii, 2011. no. 6, pp. 152-155.

11. Wang J.R., Lin S.W. Examining Reflective Thinking: A Study of Changes in Methods Students' Conceptions and Understandings of Inquiry Teaching. International Journal of Science and Mathematics Education, 2008, volume 6 , Issue 3, pp. 459-479.

12. Wulfov B. Z., Harkin, V. N. Pedagogy of reflection (p. 111). Moscow, Publisher Master. 1995.

13. Yuen Lie Lim L.A. A comparison of students' reflective thinking across different years in a problem-based learning environment. Instructional Science, 2011, Volume 39, Issue 2, pp. 171-188.

14. Bagdai E.V. Formation of the student's reflexive position in the process of studying humanities. Diss. PhD Ped. Sci., Kirov, 2007. 166 p. (in Russian)

15. Ishkov A.D. Student learning activities: psychological factors of success: a monograph. Moscow, DIA Publ., 2004. 556 p. (in Russian)

16. Karpov A.V. Reflexivity as a mental property and a method for its diagnosis. Psychological Journal. 2003. Vol. 24. no. 5. pp. 45-57. (in Russian)

17. Krasilnikova V.A. Theory and technology of computer learning and testing: a monograph. Moscow, House of Pedagogy, IPK GOUOGU Publ., 2009. 333 p. (in Russian)

18. Marushchak I.P. Pedagogical support of the formation of the reflexive position of university students through interactive learning technologies. Diss. PhD Ped. Sci., Tver, 2012. 223 p. (in Russian)

19. Osipova S.I. Designing by the student of an individual educational trajectory in the conditions of informatization of education: monograph. Moscow, INFRA Publ, 2013.140 p. (in Russian)

20. Sokolova I.Yu., Kabanov G.P. The quality of training in a technical college and technology of education: a manual for teachers, graduate students, undergraduates. Tomsk, Publishing House TGPU. 2003.203 p. (in Russian) 
21. Feklushkina A.V. Formation of reflective activity of students in the process of studying mathematics. Personality, family, society: questions of pedagogy and psychology. 2011. Part 2. (in Russian)

22. Frolov I.H. E-learning as a form of organization of educational process in the XXI century. Informatics and education. 2009. no. 2. pp. 109-110. (in Russian)

23. Khutorskoy A.V. Pedagogical innovation: studies. allowance for stud. higher studies. institutions. Moscow, Academy Publishing Center. 2010. 256 p. (in Russian)

24. Yakimanskaya I.S. Psychological foundations of mathematical education: textbook for students of pedagogical universities. Moscow: Academy Publishing Center. 2004. 320 p. (in Russian)

\section{Информация об авторах} Симонова Галина Ивановна (Россия, Киров)

Доцент, доктор педагогических наук Директор педагогического института Вятский государственный университет

E-mail: gi_simonova@vyatsu.ru

\section{Конышева Алия Вазиховна} (Россия, Киров)

Кандидат педагогических наук Доцент кафедры педагогики Вятский государственный университет E-mail: av_konysheva@vyatsu.ru

Котряхов Николай Васильевич (Россия, Киров)

Профессор, доктор педагогических наук Профессор кафедры педагогики

Вятский государственный университет
Information about the authors Galina I. Simonova

(Russia, Kirov)

Associate Professor, Doctor of Pedagogical Sciences Director of the Pedagogical Institute Vyatka State University

E-mail: gi_simonova@vyatsu.ru

\section{Aliya V. Konysheva}

(Russia, Kirov)

PhD in Pedagogical Sciences

Associate Professor of the Department of Pedagogy Vyatka State University

E-mail: av_konysheva@vyatsu.ru

Nikolay V. Kotryahov

(Russia, Kirov)

Professor, Doctor of Education

Professor of the Department of Pedagogy

Vyatka State University

\section{Ссылка для цитированиягост}

Симонова Г. И., Конышева А. В., Котряхов Н. В. Особенности формирования рефлексивной позиции студентов в процессе обучения в вузе средствами информационно-коммуникационных технологий // Перспективы науки и образования. 2018. № 6 (36). С. 65-73. doi: 10.32744/pse.2018.6.7

\section{For Reference ${ }^{\mathrm{APA}}$}

Simonova, G. I., Konysheva, A. V., Kotryakhov, N. V. (2018). Peculiarities of reflexive position formation in students within educational process at the university by information-and-communication technologies. Perspektivy nauki i obrazovania-Perspectives of Science and Education, 36 (6), 65-73. doi: 10.32744/ pse.2018.6.7. (In Russ., abstr. in Engl.) 